\title{
Arginase in Parasitic Infections: Macrophage Activation, Immunosuppression, and Intracellular Signals
}

\author{
Cinthia C. Stempin, Laura R. Dulgerian, Vanina V. Garrido, and Fabio M. Cerban
}

Inmunología, CIBICI-CONICET, Departamento de Bioquímica Clínica, Facultad de Ciencias Químicas, Universidad Nacional de Córdoba, 5000 Córdoba, Argentina

Correspondence should be addressed to Fabio M. Cerban, fcerban@fcq.unc.edu.ar

Received 31 July 2009; Accepted 30 September 2009

Academic Editor: Luis I. Terrazas

Copyright ( $\odot 2010$ Cinthia C. Stempin et al. This is an open access article distributed under the Creative Commons Attribution License, which permits unrestricted use, distribution, and reproduction in any medium, provided the original work is properly cited.

\begin{abstract}
A type 1 cytokine-dependent proinflammatory response inducing classically activated macrophages (CaM $\phi$ s) is crucial for parasite control during protozoan infections but can also contribute to the development of immunopathological disease symptoms. Type 2 cytokines such as IL-4 and IL-13 antagonize CaM $\phi$ s inducing alternatively activated macrophages (AaM $\phi$ s) that upregulate arginase-1 expression. During several infections, induction of arginase-1-macrophages was showed to have a detrimental role by limiting $\mathrm{CaM} \phi$-dependent parasite clearance and promoting parasite proliferation. Additionally, the role of arginase-1 in T cell suppression has been explored recently. Arginase- 1 can also be induced by IL-10 and transforming growth factor- $\beta$ (TGF- $\beta$ ) or even directly by parasites or parasite components. Therefore, generation of alternative activation states of macrophages could limit collateral tissue damage because of excessive type 1 inflammation. However, they affect disease outcome by promoting parasite survival and proliferation. Thus, modulation of macrophage activation may be instrumental in allowing parasite persistence and long-term host survival.
\end{abstract}

\section{Macrophage Diversity}

Macrophages are innate immune cells that play an indispensable role in the primary response to pathogens but also they play a role in the resolution of inflammation and tissue homeostasis. They can be polarized by the microenvironment to mount specific functions, as a consequence they represent a group of heterogeneous immune cells. The first indication of macrophage heterogeneity was showed by Gordon and colleagues during their studies of mannose receptor (MR) regulation in IL-4-treated $\mathrm{M} \phi$ [1]. They demonstrated that macrophage treatment with IL-4 induced an alternative activation phenotype different to the classical macrophage activation $(\mathrm{CaM} \phi)$, known to be dependent on interferon- $\gamma($ IFN- $\gamma)$ and tumor-necrosis factor (TNF) [24]. Gordon later included the effects of IL-13 in the definition of alternative activation because IL-13 shares a common receptor chain with IL-4 and exerts similar effects on macrophages [5]. However, the name "alternative activated macrophages, $(\mathrm{AaM} \phi)$ " may have led to some confusion because it implicates that it is the only other way that macrophages can be activated. In fact, Mosser and colleagues showed later that this was the case. They demonstrated that macrophage treatment with classic stimulus, like LPS or IFN- $\gamma$, in the presence of immune complexes induces the generation of a cellular type different from the $\operatorname{CaM} \phi$. These cells release large amounts of IL-10 inhibiting the IL12 synthesis. These $\mathrm{M} \phi$ have been denominated "Type $2 \mathrm{M} \phi$ " (T2a $\mathrm{M} \phi$ ) because they favor the development of type II adaptative immune response $[6,7]$.

In 1999 Goerdt and Orfanos proposed a classification of activation phenotypes based on grouping all activators other than IFN- $\gamma$ and LPS/microbes into a common alternative activation group [8]. However, nowadays the classification has been extended. It has been proposed that a categorization in which M1 polarization included classical activated macrophages, while M2 polarization, mainly associated with antiparasitic and tissue repair programs, was subdivided in M2a or alternatively activated macrophages: M2b, corresponding to type II activated macrophages and M2c, which 
includes heterogeneous macrophage deactivation stimuli such as glucocorticoids and TGF- $\beta$ [9-11]. All of these types of macrophages can be found in a variety of immunological conditions, such as immune responses to different pathogens, tumors, and autoimmune diseases. Therefore, Mosser and colleagues propose a macrophage classification based on the fundamental macrophage functions such as host defense, wound healing, and immune regulation [12]. However, it is difficult to say which way is the best for classifying them. First, there are no specific biochemical markers to assign to each population. Second, it has been demonstrated that macrophages "sequentially" change their functional phenotype in response to microenvironmental changes. Therefore, macrophages can exhibit, at the same time, different kinds of markers associated with other types of macrophages $[13,14]$. The plasticity of macrophages and high complexity of the in vivo environments result in the induction of various types of macrophages during protozoan infections and differentially affect the course of disease. Therefore, as a matter of simplicity, we will use the terms $\mathrm{CaM} \phi$ and $\mathrm{AaM} \phi$. In this review we will focus on the relevance of arginase-1, an enzyme expressed in $\mathrm{AaM} \phi$, in parasitic infection and how the arginase-1 expressing macrophages can be exploited by parasites. In addition, we will discuss the $\mathrm{T}$ cell suppressive activity of these cells and some of the complex intracellular mechanisms involved in the induction of this enzyme.

\section{Arginase-1 versus iNOS}

$\mathrm{CaM} \phi$ s have on enhanced ability to present antigens and eliminate intracellular pathogens. The antimicrobial actions have been attributed to the nitric oxide (NO) release and also to the synthesis of several products of $\mathrm{NO}$ reaction, such as peroxynitrite [15]. NO is produced from L-arginine by the inducible isoform of Nitric Oxide Sinthase (iNOS) enzyme [16]. iNOS is induced in macrophages through the inflammatory cytokines such as TNF- $\alpha$, IFN- $\gamma$, and IL-12 [17].

During infections with protozoan parasites such as Leishmania, Plasmodium, or Trypanosoma, an IFN- $\gamma$-dependent proinflammatory response triggering the development of $\mathrm{CaM} \phi \mathrm{s}$ is required to control parasitemia, especially during the acute phase of infection [18-21]. Therefore, $\mathrm{CaM} \phi \mathrm{s}$ are essential components of host defense, but their activation must be securely controlled because the cytokines and mediators that they produce can lead to host-tissue damage. On the other hand, depending on parasite virulence, host genotype and stage of infection, hosts can also produce type 2 anti-inflammatory immune responses which affect disease outcome $[22,23]$.

Type 2 cytokines such as IL-4 and IL-13 antagonize $\mathrm{CaM} \phi$ s inducing arginase-1 expression in macrophages [24], a prototypic alternative activation marker in mouse macrophages [10]. The expression of arginase-1 induces a shift of arginine metabolism from NO production via iNOS toward production of L-ornithine, a precursor for polyamines and collagen, thereby contributing to the production of the extracellular matrix important for wound healing $[8,25]$. Although the term "alternatively activated" macrophages has been indicated for IL-4 and IL-13, stimulated macrophages [10] factors such as IL-10 or TGF- $\beta$ [5] and phagocytosis of apoptotic cells [26] also antagonize $\mathrm{CaM} \phi s$ and induce alternative, non-M1 activation states in macrophages.

$\mathrm{AaM} \phi \mathrm{s}$ in normal conditions, have been identified in tissues in which it is necessary to avoid the inflammation, such as lung and placenta $[8,27]$. In addition, it was demonstrated an increase in arginase activity in peripheral blood of pregnant women and in term placenta [28]. Moreover, these macrophages have been identified during the phase of remission of acute and chronic inflammatory reactions like in psoriasis and rheumatoid arthritis [29, 30]. Therefore, generation of $\mathrm{AaM} \phi$ s after inflammation would favor the maintenance of homeostasis. Nevertheless, several evidence obtained during different infections indicate that IL-4/IL13-dependent AaM $\phi$ s exacerbate disease by limiting $\mathrm{CaM} \phi$ sdependent parasite clearance and promoting parasite proliferation $[31,32]$. Therefore, the successful elimination of an infectious agent depends on the existence of temporary and space signals that regulate the type of macrophage activation.

\section{Arginase-1 Expressing Macrophages in Helminth Infections}

Helminths are worm parasites that represent a common infectious agent in developing countries. Protection against helminths depends on IL-4 and IL-13. However, when the infection progress to a chronic stage, Th2 cytokine results in $\mathrm{AaM} \phi$ s recruitment, which leads to fibrosis and $\mathrm{T}$ cell suppression [33-36]. During Brugia malayi experimental infection, it has been observed that the IL-4-dependent macrophage recruitment actively suppress the proliferation of lymphocytes [37]. In addition, in a model of cysticercosis, Taenia crassiceps infected mice showed a Th1 response in early stages of the infection, which is replaced by a Th2 in the chronic stage [35]. In contrast to other helminths infections, $\mathrm{CaM} \phi \mathrm{s}$ are important for the clearance of $T$. crassiceps [35]. However, in chronic stages, the Th2 response results in $\mathrm{AaM} \phi$ s, that exhibit arginase activity [33]. It have been reported that carbohydrates present in $T$. crassiceps can produce the detrimental change from Th1 response towards Th2 during this infection [38]. Besides, it was demonstrated that previous infection of mice with the $T$. crassiceps favors parasitemia and induces larger cutaneous lesions during both Leishmania major and Leishmania mexicana coinfections. These authors postulated that helminth infection may facilitate Leishmania installation by inducing arginase-1 expressing macrophages [39].

On the other hand, Schistosoma mansoni infection, as well as the other helminth infection mentioned above, leads to the development of a Th2 cell response. Using IL-4 and IL-13 knockout mice, Brunet and colleagues demonstrated that the Th2 immune response is critical for the survival during acute S. mansoni infection [40, 41]. They concluded that the severity of the infection in the knockout mice was due to an increase in the TNF- $\alpha$ and NO levels. 
However, when mice are persistently infected, the chronic Th2 response becomes highly harmful contributing to the development of hepatic fibrosis and portal hypertension $[42,43]$. In addition, the Th2 response was also associated with the accumulation of a large number of arginase-1 expressing macrophages in and around the granulomas [42] and arginase- 1 expressing macrophages in peritoneal cavity and increased circulating polyamines [44]. The presence of arginase-1 expressing macrophages was originally hypothesized to be involved in promoting the collagen deposit and the fibrosis hepatic through proline synthesis mediated by arginase-1 [25]. However, Herbert and colleagues demonstrated later using mice deficient in $\mathrm{AaM} \phi$ s (Il4ra ${ }^{-/ f l o x} ;$ LysMcre) that the alternative macrophage activation is essential for survival during schistosomiasis and down modulates T helper 1 responses and immunopathology [45]. Moreover, the development of Th2-driven fibrosis in those mice S. mansoni infected appears normal [45]. Recently, it was demonstrated that macrophage-specific arginase-1 exhibits both anti-inflammatory and antifibrotic activities during Th2-driven inflammatory responses induced by S. mansoni [46]. The authors demonstrated that, instead of promoting Th2-disease, macrophage-specific arginase-1 contributes to the resolution of schistosomiasis by inhibiting CD4+ T cell effector function [46]. The data showed by Pesce and colleagues identify arginase-1-expressing macrophage as critical mediators of immune down-modulation in chronic schistosomiasis [46]. Additionally, it has been also recently demonstrated that the resistin-like molecule (RELM) $\alpha$, which is another molecule expressed by $\operatorname{AaM} \phi$, is also involved in the Th2 cytokine-down modulation during the pulmonary inflammation observed in schistosomiasis [47].

On the other hand, infection of mice with the natural mouse gastrointestinal helminth parasite Heligmosomoides polygyrus triggers a highly polarized Th2 response. Memory $\mathrm{CD} 4+\mathrm{T}$ cells rapidly accumulate at the host-parasite interface and secrete IL-4, inducing localized development of $\operatorname{AaM} \phi s$ recruited to this site. These macrophages impair larval parasite health and mobility through an arginasedependent pathway, contributing to eventual expulsion of adult worms [48]. It has been demonstrated that Th2 responses enhance gut contractility and luminal fluid secretion, leading to worm expulsion $[49,50]$. Furthermore, specific inhibition of arginase activity during the infection with intestinal nematode parasite Nippostrongylus brasiliensis interfered with smooth muscle contractility and affected partially the protective immunity of the host [51]. Therefore, arginase- 1 expressing macrophages are widely induced by helminths and they play different roles in those infections.

\section{Arginase in Protozoan Infections}

The type 1 pro-inflammatory response, crucial for parasite control during the acute phase of infection with protozoan parasites, should be carefully balanced by anti-inflammatory molecules like IL-10 and TGF- $\beta$ to avoid excessive collateral damage to host tissues and cells. However, in many cases protozoan parasites take advantage of this anti-inflammatory response to persist in the host. It has been reported that during Trypanosoma brucei infection, sustained $\mathrm{CaM} \phi \mathrm{s}$ could induce tissue damage, therefore mice that progress to a chronic stage, generate a Th2 response, able to favor the development of $\mathrm{AaM} \phi$ [52]. In addition, it has been observed that Leishmania major has the ability to inhibit the production of IL-12 through the induction of IL-10 and TGF- $\beta$ [53]. This imbalance underlies a shift from a Th1 to a Th2 immune response and reflects the susceptibility to the disease. This environment generates $\mathrm{AaM} \phi$ facilitating the dissemination of the parasite in the host $[54,55]$. Macrophage-IL-4 treatment previous to the infection with Leishmania major or Leishmania infantum biases the metabolism of L-arginine through arginase-1 towards the synthesis of polyamines, which promote the intracellular growth of the parasite [56]. Also, macrophage arginase-1-inhibition reduced the growth of the parasites due to an increase in NO production [56]. Additionally, in a mouse model of L. major infection, arginase- 1 is induced in both susceptible and resistant mice during the development of the disease. The induction of the host arginase- 1 in both strains is mediated by the balance between IL-4 and IL-12 and opposite to iNOS expression. Moreover, inhibition of arginase-1 reduces the number of parasites and delays disease outcome in susceptible mice, while treatment with L-ornithine increases the susceptibility of the resistant mice [54]. Furthermore, Kropf and colleagues also demonstrated that arginase-1 activity and production of polyamines are key factors in the regulation of leishmaniasis [55]. Moreover, another indication for the contribution of $\mathrm{AaM} \phi$ to Leishmania susceptibility was demonstrated using macrophage/neutrophil-specific IL$4 \mathrm{R} \alpha$-deficient mice. Those mice showed a significantly delayed disease progression with normal Th2 and type 2 antibody responses but improved macrophage leishmanicidal effector functions and reduced arginase-1 activity [57]. Therefore, arginase- 1 induction could be considered a marker of disease in leishmaniasis.

On the other hand, a Th1 immune response, involving IL-12, IL-18, and IFN- $\gamma$ as well as NO-producing $\operatorname{CaM} \phi$, favors the control of the early Trypanosoma cruzi infection that causes Chagas disease [58, 59]. However, if uncontrolled, the Th1 immune response causes pathology. In this respect, IL-4 and IL-10 have been implicated in resistance to $T$. cruzi by balancing the early stage proinflammatory immune environment $[60,61]$. Therefore, once again, the Th2 environment could induce $\mathrm{AaM} \phi$ s and favor the installation of the parasite in the host. Arginase-1 activity, nevertheless, has not been necessarily induced by IL- 4 during $T$. cruzi infection. We have demonstrated arginase-1 induction by the parasite component, cruzipain $[62,63]$. We have showed that mice immunization with the immunodominant antigen cruzipain results in high Th2 cytokine secretion (IL4 , IL-5, IL-10), and marginal levels of IFN- $\gamma$ and IL-12 [62]. In addition, cruzipain-stimulated macrophages exhibit increased arginase-1 activity and expression, and secrete IL-10 and TGF- $\beta[63,64]$. Moreover, cruzipain-activated macrophages favor T. cruzi growth at the same extend as was observed in IL-4-treated macrophages [63] and arginase-1inhibition leads to a drastic reduction in T. cruzi replication 
in macrophages [64]. Additionally, we found an increase in arginase-1 activity during acute infection in BALB/c mice. Furthermore, arginase- 1 inhibition leads to a reduction in the number of amastigotes in infected macrophages (Stempin, et al. unpublished data). Therefore, arginase1 induction could favor Trypanosoma cruzi persistence in the host as was observed during Leishmania infection. In addition, it was demonstrated that peripheral blood monocytes of $T$. cruzi infected rats are not able to produce NO due to an increase in the arginase-1 activity [65]. The emergence of arginase-1-expressing macrophages is further promoted by the ingestion of apoptotic cells generated by NO during the early stage of T. cruzi infection, and the concomitant enhanced TGF- $\beta$ secretion [26]. Therefore, this results in a decreased oxidative burst and persistent parasite growth in the infected macrophages because of the down regulation of iNOS activity. Moreover, TGF$\beta$, by inducing ornithine decarboxylase activity, favors the synthesis of polyamines that are essential for the intracellular parasite replication [66]. Hence, T. cruzi might evade the host-protective Th1 immune response mediated by $\mathrm{CaM} \phi \mathrm{s}$ by instructing Th2 cytokine secretion, which stimulates $\operatorname{AaM} \phi$. Besides down regulating the inflammatory immune response, $\mathrm{AaM} \phi$ s might favor the onset of a chronic infection during Chagas disease.

\section{Alternative Activation Markers in Human and Murine Macrophages}

$\operatorname{AaM} \phi$ generation is associated with changes in macrophage function and distinctive gene signatures. Although the alternative activation markers for human and murine macrophages have long been considered analogous, there are differences between species. IL-4 and IL-13 induce genes conserved in both species such as MHC-II, MR, MGL1/2, and CD23 as well as divergent ones such as arginase-1, chitinase-like molecules (Ym1/2), and RELM $\alpha$ in mouse, and nucleotide G protein-coupled receptors (GPCRs) in humans [10, 67-69]

As mentioned before, mouse arginase- 1 is a prototypic alternative activation marker and its induction is confined mainly to murine macrophages [70]. However, it was recently demonstrated that human mononuclear cells can adopt an alternative activation profile during the infection with the filarial nematode parasite Wuchereria bancrofti, the etiological agent of lymphatic filariasis [71]. The authors demonstrated that these cells express the arginase encoding genes and a member of the resistin family [71]. Under the appropriate environment, this outcome would indicate that human monocytes can undergo alternative activation, similar to that found for mouse macrophages [72].

In addition, it was demonstrated that arginase- 1 is expressed constitutively in human granulocytes and would participate like a new effector mechanism against fungi, decreasing arginine availability in phagolysosome [73]. In contrast to murine arginase-1, human granulocyte arginase1 expression is not influenced by proinflammatory or antiinflammatory cytokines in vitro. On the other hand, its location is not cytosolic but it is present in azurophils granules and it was determined that human arginase-1 present in neutrophils has the capacity to suppress $\mathrm{T}$ cells [74].

Although the general functions and behaviors of murine and human $\mathrm{AaM} \phi$ s are thought to be preserved, the genes required for such functions are to some extent different.

The divergences between species and their functional repercussion require further investigation [10].

\section{Role of Arginase-1 in Immunosuppression}

The ability of arginase-1expressing macrophages to suppress $\mathrm{T}$ cell proliferation could explain their presence in placenta and lung of healthy individuals $[8,75]$. However, the presence of suppressive arginase- 1 expressing macrophages during infections as well as during cancer could be detrimental for the host [76].

As was mentioned above, during Brugia malayi experimental infection, it has been demonstrated that IL-4dependent macrophages recruited in vivo actively suppress the proliferation of lymphocytes. These macrophages block proliferation by cell-to-cell contact, implicating a receptormediated mechanism. [37]. In addition, it was also recently demonstrated that arginase-1 plays an essential role in the $\mathrm{T}$ cell suppression observed at the site of pathology in nonhealing leishmaniasis lesions [77]. Modollel and colleagues demonstrated that arginase- 1 is highly expressed at the site of pathology in nonhealing lesions and causes local depletion of L-arginine which impairs the capacity of T cells in the lesion to proliferate and to produce IFN- $\gamma$, required for parasite killing. They clearly showed that arginase- 1 is mediating Tcell immunosuppression since its inhibition or the injection of L-arginine reverses suppression and results in macrophage efficient control of parasite replication [77]. However, during schistosomiasis, macrophage-specific arginase- 1 contributes to the resolution of the infection by inhibiting CD4+ $\mathrm{T}$ cell proliferation and cytokine production. The authors showed that arginine depletion by arginase-1 expressing macrophages was the primary suppressive mechanism while no significant contribution for IL-10 or TGF- $\beta$ was observed in these studies [46]. Moreover, Terrazas and colleagues have showed that Taenia crassiceps-induced macrophages have suppressive $\mathrm{T}$ cell activity and this effect was IL-10, IFN- $\gamma$, NO independent, and cell-cell contact dependent [36]. Taenia crassiceps-induced macrophages showed an alternative activated profile with high expression of Fizz1, Ym1, and arginase-1 [36]. The authors demonstrated that the suppressive ability of the Taenia crassiceps-induced macrophages involves programmed death ligand molecules, PD-L1 and PD-L2, which were found to be upregulated in those macrophages [36]. These molecules bind to the receptor programmed death 1 (PD-1) which is expressed on activated T cells ( $\mathrm{B}$ cells and myeloid cells also) and their interactions result in down-modulation of $\mathrm{T}$ cell responses $[78,79]$. In addition, the selective upregulation of PD-L1 on macrophages by Schistosoma mansoni worms has shown to induce anergy of $\mathrm{T}$ cells during early acute stages of infection 
before the subsequent emergence of egg-induced $\mathrm{T}$ cell suppression in the chronic stages of infection [80]. Studies in progress in our group demonstrated that the PD-1/PD$\mathrm{L}$ pathway may be also involved in the immunosuppression observed during the acute phase of T. cruzi infection [81]. We observed change in the expression of these molecules during the acute phase of T. cruzi infection in CD3+, $\mathrm{CD} 11 \mathrm{c}+$, and F4/80+ peritoneal cells. In addition, T. cruzimacrophages were able to suppress concanavalin A (ConA) $\mathrm{T}$ cell induced proliferation which is restored by blocking PD-1 pathway with antibodies or by inhibiting arginase-1 activity. In addition, our preliminary data suggest that PD-L2 could be involved in arginase-1 induction in T. cruzi infected macrophages (Dulgerian et al. unpublished data). Therefore, this may suggest that PD-1-PD-Ls interaction is important in arginase-1 modulation and in T. cruzi replication in macrophages.

In this context, it has been recently showed that the activity and expression of arginase- 1 could be regulated by both PD-1 and CTLA-4 on the myeloid-derived suppressor cells (MDSCs) [82]. It has been extensively demonstrated that increased metabolism of L-arginine by myeloid cells can result in the impairment of lymphocyte responses to antigen during immune responses and tumor growth [76]. PD-1 molecules expressed on MDSCs can be involved in the regulation of MDSC function by regulating the activation and expression of arginase- 1 . The regulation of arginase1 activity and expression by costimulatory or co-inhibitory molecules on the MDSCs might be important in inducing and maintaining of immunosuppression [76].

On the other hand, the role of human granulocyte arginase in immunosuppression was explored by Munder and colleagues [74]. They demonstrated that upon human polymorphonuclear granulocytes (PMN) cell death, arginase is liberated and very high activities of this enzyme accumulate extracellularly during purulent inflammatory reactions. PMN arginase induces a profound suppression of $\mathrm{T}$ cell proliferation and cytokine synthesis. This T cell suppression is due to arginase-mediated depletion of L-arginine in the $\mathrm{T}$ cell environment, which leads to $\mathrm{CD} 3 \zeta$ chain downregulation [74]. Recently they showed that PMN arginasemediated suppression reach also NK cells [83]. Granulocyte arginase severely impairs NK cell proliferation and IL-12/IL18-induced secretion of IFN- $\gamma[83]$.

\section{Signals Involved in Arginase-1 Induction}

One of the first works that investigated about the intracellular signals involved in arginase induction by LPS showed that protein kinase A (PKA) activation was required whereas the activation of the enzyme did not depend on PKC, neither the intracellular levels of $\mathrm{Ca}++$ [84]. Later, the signals involved in IL-13 arginase-1-induction were studied by Chang and colleagues [85]. They demonstrated that IL-13 down-regulates NO production through arginase induction via cAMP/PKA, tyrosine kinase (TK), and p38 MAPK signaling [85]. Recently findings correlate with the previous role of PKA in arginase induction in macrophages [86]. We have previously shown that arginase-1 induction mediated by cruzipain involves the activation of multiple intracellular signals such as TK, PKA, and p38 MAPK [64, 87], similar to the signals triggered by IL-13 [85]. Furthermore, it was demonstrated that the transcription factor STAT-6 is required for arginase-1 induction in IL-4 and IL-13-treated macrophages $[88,89]$. However, it was recently shown that some intracellular pathogens induced arginase-1 expression in mouse macrophages through the Toll Like Receptor (TLR) pathway [90]. In contrast to diseases dominated by Th2 responses in which arginase- 1 expression is greatly increased by IL- 4 and IL-13 signaling through the transcription factor STAT6, TLR-mediated arginase-1 induction was independent of the STAT6 pathway. Specific elimination of arginase1 in macrophage favored host survival during Toxoplasma gondii infection and decreased lung bacterial load during tuberculosis infection. Therefore, TLR pathway could be also advantageous to certain pathogens [90].

\section{Concluding Remarks}

The diversity of macrophage activation states is probably as diverse as the number of environmental conditions to provide the appropriate immune response. It is influenced by the complexity of the stimulus, the sequence of exposure, and the status of macrophage differentiation at the time of exposure. In this review we have focused our discussion on the role of arginase-1-macrophages in parasitic infections. The outcome of protozoan infections is crucially dependent on the order, timing, and relative strength of type 1 versus type 2 responses and $\mathrm{CaM} \phi$ versus $\mathrm{AaM} \phi$ generation (Figure 1). During several infections induction of arginase1- macrophages was showed to have a detrimental role by limiting CaM $\phi$-dependent parasite clearance and promoting parasite proliferation. Additionally, the role of arginase in T-cell suppression has been explored recently. During several infections, arginase- $1 \mathrm{~T}$ cell suppression contributes to failure to eliminate the pathogen. However, during schistosomiasis arginase-1-expressing macrophages suppress Th2-dependent inflammation and fibrosis, contributing to the resolution of the infection. Arginase suppressive activity has been mainly associated with L-arginine depletion leading to $\mathrm{CD} 3$ instability. In addition, PD-1 and their ligands have been also involved in arginase-1 suppression (Figure 2). On the other hand, although arginase-1 induction is confined mainly to murine macrophages, it was demonstrated that it is expressed constitutively in human granulocytes and it showed some similar function to the murine arginase (Figure 2). However, although the general functions and behaviors of murine and human $\mathrm{AaM} \phi$ s are thought to be preserved, human alternative activated macrophages should be further investigated.

Additionally, recent findings have improved the understanding of signals involved in arginase-1 expression. In addition to the well-known role of STAT-6 in IL-4 and IL13-arginase induction, during Th1 responses, a novel TLR dependent but STAT6-independent pathway of arginase-1 expression was identified. 


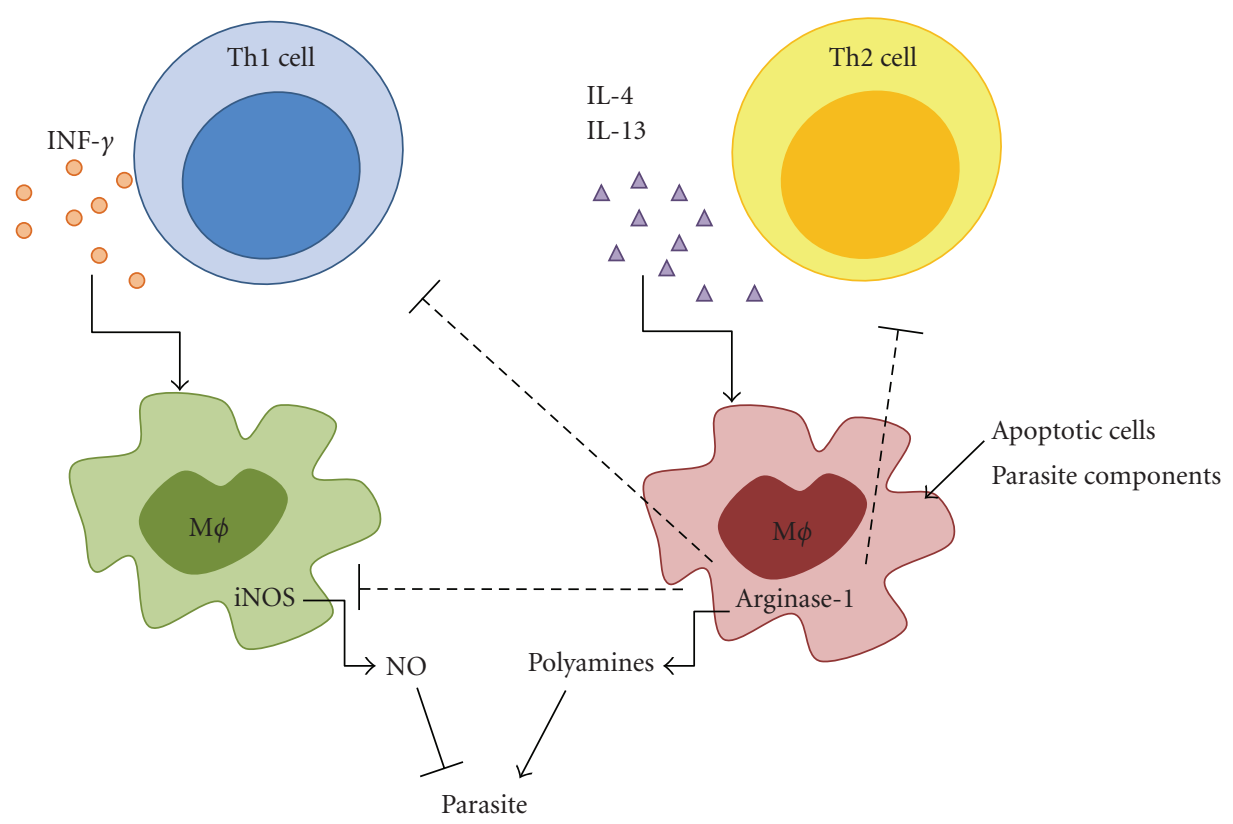

FIGURE 1: Role of macrophage activation in parasitic infections. A type 1 cytokine-dependent proinflammatory response inducing classically activated macrophages $(\mathrm{CaM} \phi \mathrm{s})$ leads to NO production and also to the synthesis of several products of NO reaction. CaM $\phi$ s are crucial for parasite control during protozoan infections but can also contribute to the development of immunopathological disease symptoms. Type 2 cytokines such as IL- 4 and IL-13 antagonize CaM $\phi$ s inducing alternatively activated macrophages (AaM $\phi$ s) that upregulate arginase-1 expression. Arginase-1 can also be induced during the infection by apoptotic cells or even directly by parasites or parasite components. Arginase-1 limits $\mathrm{CaM} \phi$-dependent parasite clearance promoting parasite proliferation. Additionally, arginase- 1 suppresses $\mathrm{T}$ cell response. Therefore, generation of alternative activation states of macrophages could limit collateral tissue damage because of excessive type 1 inflammation. However, they affect disease outcome by promoting parasite survival and proliferation.

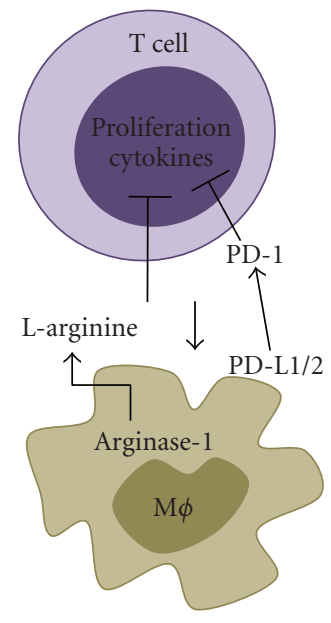

Mouse

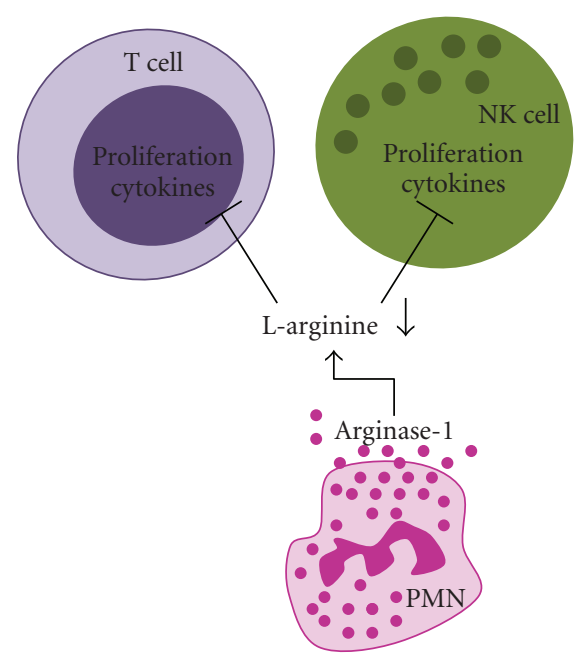

Human

FIgURE 2: Role of arginase in immunosuppression. During parasitic infections, mouse macrophage-specific arginase-1 induces $\mathrm{T}$ cell suppression due to arginase-mediated depletion of L-arginine in the T cell environment, which leads to CD3 $\zeta$ chain down-regulation. Additionally, PD-1/PD-L pathway may be also involved in the T cell suppression. Furthermore, human granulocyte arginase can also induce immunosuppression by a similar mechanism. Upon human polymorphonuclear granulocytes (PMN) cell death, arginase is liberated and high activities of this enzyme accumulate extracellularly during purulent inflammatory reactions. PMN arginase induces a profound suppression of $\mathrm{T}$ cell and NK cell proliferation and cytokine synthesis. 
Finally, our knowledge of how different types of macrophages affect the course of infectious diseases caused by protozoan parasites should provide therapeutic benefits in controlling these infections.

\section{Acknowledgments}

This work was supported by Consejo Nacional de Investigaciones Científicas y Técnicas (CONICET), Secretaria de Ciencia y Tecnología de la Universidad Nacional de Córdoba (SECyT-UNC), Agencia Córdoba Ciencia (ACC), and Agencia Nacional de Promoción Científica y Tecnológica (ANPCyT). F.M.C is a permanent research fellow of CONICET. C.C.S, L.R.D, and V.V.G thank CONICET for their fellowship grants.

\section{References}

[1] M. Stein, S. Keshav, N. Harris, and S. Gordon, "Interleukin 4 potently enhances murine macrophage mannose receptor activity: a marker of alternative immunologic macrophage activation," Journal of Experimental Medicine, vol. 176, no. 1, pp. 287-292, 1992.

[2] D. M. Mosser, "The many faces of macrophage activation," Journal of Leukocyte Biology, vol. 73, no. 2, pp. 209-212, 2003.

[3] C. Nathan, "Mechanisms and modulation of macrophage activation," Behring Institut Mitteilungen, no. 88, pp. 200-207, 1991.

[4] D. K. Dalton, S. Pitts-Meek, S. Keshav, I. S. Figari, A. Bradley, and T. A. Stewart, "Multiple defects of immune cell function in mice with disrupted interferon- $\gamma$ genes," Science, vol. 259, no. 5102, pp. 1739-1742, 1993.

[5] S. Gordon, "Alternative activation of macrophages," Nature Reviews Immunology, vol. 3, no. 1, pp. 23-35, 2003.

[6] F. S. Sutterwala, G. J. Noel, P. Salgame, and D. M. Mosser, "Reversal of proinflammatory responses by ligating the macrophage Fcy receptor type I," Journal of Experimental Medicine, vol. 188, no. 1, pp. 217-222, 1998.

[7] C. F. Anderson and D. M. Mosser, "Cutting edge: biasing immune responses by directing antigen to macrophage $\mathrm{Fc} \gamma$ receptors," The Journal of Immunology, vol. 168, no. 8, pp. 3697-3701, 2002.

[8] S. Goerdt and C. E. Orfanos, "Other functions, other genes: alternative activation of antigen-presenting cells," Immunity, vol. 10, no. 2, pp. 137-142, 1999.

[9] A. Mantovani, S. Sozzani, M. Locati, P. Allavena, and A. Sica, "Macrophage polarization: tumor-associated macrophages as a paradigm for polarized M2 mononuclear phagocytes," Trends in Immunology, vol. 23, no. 11, pp. 549-555, 2002.

[10] F. O. Martinez, L. Helming, and S. Gordon, "Alternative activation of macrophages: an immunologic functional perspective," Annual Review of Immunology, vol. 27, pp. 451-483, 2009.

[11] F. O. Martinez, A. Sica, A. Mantovani, and M. Locati, "Macrophage activation and polarization," Frontiers in Bioscience, vol. 13, no. 2, pp. 453-461, 2008.

[12] D. M. Mosser and J. P. Edwards, "Exploring the full spectrum of macrophage activation," Nature Reviews Immunology, vol. 8, no. 12, pp. 958-969, 2008.

[13] R. D. Stout, C. Jiang, B. Matta, I. Tietzel, S. K. Watkins, and J. Suttles, "Macrophages sequentially change their functional phenotype in response to changes in microenvironmental influences," The Journal of Immunology, vol. 175, no. 1, pp. 342-349, 2005.

[14] K. J. Mylonas, M. G. Nair, L. Prieto-Lafuente, D. Paape, and J. E. Allen, "Alternatively activated macrophages elicited by helminth infection can be reprogrammed to enable microbial killing," The Journal of Immunology, vol. 182, no. 5, pp. 30843094, 2009.

[15] C. Bogdan, "Nitric oxide and the immune response," Nature Immunology, vol. 2, no. 10, pp. 907-916, 2001.

[16] J. MacMicking, Q.-W. Xie, and C. Nathan, "Nitric oxide and macrophage function," Annual Review of Immunology, vol. 15, pp. 323-350, 1997.

[17] M. Modolell, I. M. Corraliza, F. Link, G. Soler, and K. Eichmann, "Reciprocal regulation of the nitric oxide synthasearginase balance in mouse bone marrow-derived macrophages by TH1 and TH2 cytokines," European Journal of Immunology, vol. 25, no. 4, pp. 1101-1104, 1995.

[18] P. Kropf, M. A. Freudenberg, M. Modolell, et al., "Tolllike receptor 4 contributes to efficient control of infection with the protozoan parasite Leishmania major," Infection and Immunity, vol. 72, no. 4, pp. 1920-1928, 2004.

[19] G. Peluffo, L. Piacenza, F. Irigoín, M. N. Alvarez, and R. Radi, "L-arginine metabolism during interaction of Trypanosoma cruzi with host cells," Trends in Parasitology, vol. 20, no. 8, pp. 363-369, 2004.

[20] M. Plebanski and A. V. S. Hill, "The immunology of malaria infection," Current Opinion in Immunology, vol. 12, no. 4, pp. 437-441, 2000.

[21] M. Walther, J. Woodruff, F. Edele, et al., "Innate immune responses to human malaria: heterogeneous cytokine responses to blood-stage Plasmodium falciparum correlate with parasitological and clinical outcomes," The Journal of Immunology, vol. 177, no. 8, pp. 5736-5745, 2006.

[22] W. Noël, Gh. Hassanzadeh, G. Raes, et al., "Infection stagedependent modulation of macrophage activation in Trypanosoma congolense-resistant and -susceptible mice," Infection and Immunity, vol. 70, no. 11, pp. 6180-6187, 2002.

[23] F. M. Omer, J. B. de Souza, and E. M. Riley, "Differential induction of TGF- $\beta$ regulates proinflammatory cytokine production and determines the outcome of lethal and nonlethal Plasmodium yoelii infections," The Journal of Immunology, vol. 171, no. 10, pp. 5430-5436, 2003.

[24] M. Munder, K. Eichmann, and M. Modolell, "Alternative metabolic states in murine macrophages reflected by the nitric oxide synthase/arginase balance: competitive regulation by $\mathrm{CD}^{+}{ }^{+} \mathrm{T}$ cells correlates with Th1/Th2 phenotype," The Journal of Immunology, vol. 160, no. 11, pp. 5347-5354, 1998.

[25] M. Hesse, M. Modolell, A. C. La Flamme, et al., "Differential regulation of nitric oxide synthase- 2 and arginase- 1 by type $1 /$ type 2 cytokines in vivo: granulomatous pathology is shaped by the pattern of L-arginine metabolism," The Journal of Immunology, vol. 167, no. 11, pp. 6533-6544, 2001.

[26] C. G. Freire-De-Lima, D. O. Nascimento, M. B. P. Soares, et al., "Uptake of apoptotic cells drives the growth of a pathogenic trypanosome in macrophages," Nature, vol. 403, no. 6766, pp. 199-203, 2000.

[27] B. Mues, D. Langer, G. Zwadlo, and C. Sorg, "Phenotypic characterization of macrophages in human term placenta," Immunology, vol. 67, no. 3, pp. 303-307, 1989.

[28] P. Kropf, D. Baud, S. E. Marshall, et al., "Arginase activity mediates reversible $\mathrm{T}$ cell hyporesponsiveness in human pregnancy," European Journal of Immunology, vol. 37, no. 4, pp. 935-945, 2007. 
[29] N. Djemadji-Oudjiel, S. Goerdt, V. Kodelja, M. Schmuth, and C. E. Orfanos, "Immunohistochemical identification of type II alternatively activated dendritic macrophages (RM 3/1 ${ }^{+++}$, MS- $\left.1^{ \pm}, 25 \mathrm{~F}^{-}\right)$in psoriatic dermis," Archives of Dermatological Research, vol. 288, no. 12, pp. 757-764, 1996.

[30] S. Goerdt, R. Bhardwaj, and C. Sorg, "Inducible expression of MS-1 high-molecular-weight protein by endothelial cells of continuous origin and by dendritic cells/macrophages in vivo and in vitro," American Journal of Pathology, vol. 142, no. 5, pp. 1409-1422, 1993.

[31] W. Noël, G. Raes, G. H. Ghassabeh, P. De Baetselier, and A. Beschin, "Alternatively activated macrophages during parasite infections," Trends in Parasitology, vol. 20, no. 3, pp. 126-133, 2004.

[32] G. Raes, A. Beschin, G. H. Ghassabeh, and P. De Baetselier, "Alternatively activated macrophages in protozoan infections," Current Opinion in Immunology, vol. 19, no. 4, pp. 454-459, 2007.

[33] L. Brys, A. Beschin, G. Raes, et al., "Reactive oxygen species and 12/15-lipoxygenase contribute to the antiproliferative capacity of alternatively activated myeloid cells elicited during helminth infection," The Journal of Immunology, vol. 174, no. 10, pp. 6095-6104, 2005.

[34] J. L. Reyes and L. I. Terrazas, "The divergent roles of alternatively activated macrophages in helminthic infections," Parasite Immunology, vol. 29, no. 12, pp. 609-619, 2007.

[35] M. Rodríguez-Sosa, A. R. Satoskar, R. Calderón, et al., "Chronic helminth infection induces alternatively activated macrophages expressing high levels of CCR5 with low interleukin-12 production and Th2-biasing ability," Infection and Immunity, vol. 70, no. 7, pp. 3656-3664, 2002.

[36] L. I. Terrazas, D. Montero, C. A. Terrazas, J. L. Reyes, and M. Rodríguez-Sosa, "Role of the programmed Death-1 pathway in the suppressive activity of alternatively activated macrophages in experimental cysticercosis," International Journal for Parasitology, vol. 35, no. 13, pp. 1349-1358, 2005.

[37] P. Loke, A. S. MacDonald, A. Robb, R. M. Maizels, and J. E. Allen, "Alternatively activated macrophages induced by nematode infection inhibit proliferation via cell-to-cell contact," European Journal of Immunology, vol. 30, no. 9, pp. 2669-2678, 2000.

[38] S. Dissanayake, N. Khan, A. Shahin, S. Wijesinghe, and M. Lukic, "Induction of immunoglobulin G1, interleukin-6 and interleukin- 10 by Taenia crassiceps metacestode carbohydrates," Immunology, vol. 107, no. 4, pp. 411-419, 2002.

[39] M. Rodríguez-Sosa, I. Rivera-Montoya, A. Espinoza, et al., "Acute cysticercosis favours rapid and more severe lesions caused by Leishmania major and Leishmania mexicana infection, a role for alternatively activated macrophages," Cellular Immunology, vol. 242, no. 2, pp. 61-71, 2006.

[40] L. R. Brunet, M. Beall, D. W. Dunne, and E. J. Pearce, "Nitric oxide and the Th2 response combine to prevent severe hepatic damage during Schistosoma mansoni infection," The Journal of Immunology, vol. 163, no. 9, pp. 4976-4984, 1999.

[41] L. R. Brunet, F. D. Finkelman, A. W. Cheever, M. A. Kopf, and E. J. Pearce, "IL-4 protects against TNF- $\alpha$-mediated cachexia and death during acute schistosomiasis," The Journal of Immunology, vol. 159, no. 2, pp. 777-785, 1997.

[42] E. J. Pearce and A. S. MacDonald, "The immunobiology of schistosomiasis," Nature Reviews Immunology, vol. 2, no. 7, pp. 499-511, 2002.

[43] T. A. Wynn, R. W. Thompson, A. W. Cheever, and M. M. Mentink-Kane, "Immunopathogenesis of schistosomiasis," Immunological Reviews, vol. 201, pp. 156-167, 2004.
[44] O. M. S. Abdallahi, H. Bensalem, R. Augier, M. Daigana, M. De Reggi, and B. Gharib, "Arginase expression in peritoneal macrophages and increase in circulating polyamine levels in mice infected with Schistosoma mansoni," Cellular and Molecular Life Sciences, vol. 58, no. 9, pp. 1350-1357, 2001.

[45] D. R. Herbert, C. Hölscher, M. Mohrs, et al., "Alternative macrophage activation is essential for survival during schistosomiasis and downmodulates $\mathrm{T}$ helper 1 responses and immunopathology," Immunity, vol. 20, no. 5, pp. 623-635, 2004.

[46] J. T. Pesce, T. R. Ramalingam, M. M. Mentink-Kane, et al., "Arginase-1-expressing macrophages suppress Th2 cytokinedriven inflammation and fibrosis," PLoS Pathogens, vol. 5, no. 4, Article ID e1000371, 15 pages, 2009.

[47] M. G. Nair, Y. Du, J. G. Perrigoue, et al., "Alternatively activated macrophage-derived RELM- $\alpha$ is a negative regulator of type 2 inflammation in the lung," Journal of Experimental Medicine, vol. 206, no. 4, pp. 937-952, 2009.

[48] R. M. Anthony, J. F. Urban, F. Alem, et al., "Memory TH2 cells induce alternatively activated macrophages to mediate protection against nematode parasites," Nature Medicine, vol. 12, no. 8, pp. 955-960, 2006.

[49] A. Zhao, J. McDermott, J. F. Urban Jr., et al., "Dependence of IL-4, IL-13, and nematode-induced alterations in murine small intestinal smooth muscle contractility on Stat6 and enteric nerves," The Journal of Immunology, vol. 171, no. 2, pp. 948-954, 2003.

[50] T. Shea-Donohue, C. Sullivan, F. D. Finkelman, et al., "The role of IL-4 in Heligmosomoides polygyrus-induced alterations in murine intestinal epithelial cell function," The Journal of Immunology, vol. 167, no. 4, pp. 2234-2239, 2001.

[51] A. Zhao, J. F. Urban Jr., R. M. Anthony, et al., "Th2 cytokineinduced alterations in intestinal smooth muscle function depend on alternatively activated macrophages," Gastroenterology, vol. 135, no. 1, pp. 217-225, 2008.

[52] P. Baetselier, B. Namangala, W. Noël, L. Brys, E. Pays, and A. Beschin, "Alternative versus classical macrophage activation during experimental African trypanosomosis," International Journal for Parasitology, vol. 31, no. 5-6, pp. 575-587, 2001.

[53] T. Scharton-Kersten, L. C. C. Afonso, M. Wysocka, G. Trinchieri, and P. Scott, "IL-12 is required for natural killer cell activation and subsequent $\mathrm{T}$ helper 1 cell development in experimental Leishmaniasis," The Journal of Immunology, vol. 154, no. 10, pp. 5320-5330, 1995.

[54] V. Iniesta, J. Carcelén, I. Molano, et al., "Arginase I induction during Leishmania major infection mediates the development of disease," Infection and Immunity, vol. 73, no. 9, pp. 60856090, 2005.

[55] P. Kropf, J. M. Fuentes, E. Fähnrich, et al., "Arginase and polyamine synthesis are key factors in the regulation of experimental leishmaniasis in vivo," The FASEB Journal, vol. 19, no. 8, pp. 1000-1002, 2005.

[56] V. Iniesta, L. C. Gómez-Nieto, and I. Corraliza, "The inhibition of arginase by $N^{\omega}$-hydroxy-L-arginine controls the growth of Leishmania inside macrophages," Journal of Experimental Medicine, vol. 193, no. 6, pp. 777-783, 2001.

[57] C. Hëlscher, B. Arendse, A. Schwegmann, E. Myburgh, and F. Brombacher, "Impairment of alternative macrophage activation delays cutaneous leishmaniasis in nonhealing BALB/c mice," The Journal of Immunology, vol. 176, no. 2, pp. 11151121, 2006.

[58] V. Michailowsky, N. M. Silva, C. D. Rocha, L. Q. Vieira, J. Lannes-Vieira, and R. T. Gazzinelli, "Pivotal role of interleukin-12 and interferon- $\gamma$ axis in controlling tissue 
parasitism and inflammation in the heart and central nervous system during Trypanosoma cruzi infection," American Journal of Pathology, vol. 159, no. 5, pp. 1723-1733, 2001.

[59] M. M. Rodrigues, M. Ribeiräo, and S. B. Boscardin, "CD4 Th1 but not Th2 clones efficiently activate macrophages to eliminate Trypanosoma cruzi through a nitric oxide dependent mechanism," Immunology Letters, vol. 73, no. 1, pp. 43-50, 2000.

[60] I. A. Abrahamsohn, A. P. G. da Silva, and R. L. Coffman, "Effects of interleukin-4 deprivation and treatment on resistance to Trypanosoma cruzi," Infection and Immunity, vol. 68, no. 4, pp. 1975-1979, 2000.

[61] C. Hëlscher, M. Mohrs, W. J. Dai, et al., “Tumor necrosis factor alpha-mediated toxic shock in Trypanosoma cruzi- infected interleukin 10-deficient mice," Infection and Immunity, vol. 68, no. 7, pp. 4075-4083, 2000.

[62] L. Giordanengo, N. Guiñazú, C. Stempin, R. Fretes, F. Cerbán, and S. Gea, "Cruzipain, a major Trypanosoma cruzi antigen, conditions the host immune response in favor of parasite," European Journal of Immunology, vol. 32, no. 4, pp. 1003-1011, 2002.

[63] C. Stempin, L. Giordanengo, S. Gea, and F. Cerbán, "Alternative activation and increase of Trypanosoma cruzi survival in murine macrophages stimulated by cruzipain, a parasite antigen," Journal of Leukocyte Biology, vol. 72, no. 4, pp. 727$734,2002$.

[64] C. C. Stempin, T. B. Tanos, O. A. Coso, and F. M. Cerbán, "Arginase induction promotes Trypanosoma cruzi intracellular replication of Cruzipain-treated J774 cells through the activation of multiple signaling pathways," European Journal of Immunology, vol. 34, no. 1, pp. 200-209, 2004.

[65] D. L. Fabrino, L. L. Leon, G. G. Parreira, M. Genestra, P. E. Almeida, and R. C. N. Melo, "Peripheral blood monocytes show morphological pattern of activation and decreased nitric oxide production during acute Chagas' disease in rats," Nitric Oxide, vol. 11, no. 2, pp. 166-174, 2004.

[66] L. Piacenza, G. Peluffo, and R. Radi, "L-arginine-dependent suppression of apoptosis in Trypanosoma cruzi: contribution of the nitric oxide and polyamine pathways," Proceedings of the National Academy of Sciences of the United States of America, vol. 98, no. 13, pp. 7301-7306, 2001.

[67] P. Loke, M. G. Nair, J. Parkinson, D. Guiliano, M. Blaxter, and J. E. Allen, "IL-4 dependent alternatively-activated macrophages have a distinctive in vivo gene expression phenotype," BMC Immunology, vol. 3, article 7, pp. 1-11, 2002.

[68] G. Raes, P. De Baetselier, W. Noë;l, A. Beschin, F. Brombacher, and H. G. Gholamreza, "Differential expression of FIZZ1 and Ym1 in alternatively versus classically activated macrophages," Journal of Leukocyte Biology, vol. 71, no. 4, pp. 597-602, 2002.

[69] G. Raes, W. Noël, A. Beschin, L. Brys, P. De Baetselier, and Gh. Hassanzadeh, "FIZZ1 and Ym as tools to discriminate between differentially activated macrophages," Developmental Immunology, vol. 9, no. 3, pp. 151-159, 2002.

[70] G. Raes, R. Van den Bergh, P. De Baetselier, et al., "Arginase-1 and $\mathrm{Ym} 1$ are markers for murine, but not human, alternatively activated myeloid cells," The Journal of Immunology, vol. 174, no. 11, pp. 6561-6562, 2005.

[71] S. Babu, V. Kumaraswami, and T. B. Nutman, "Alternatively activated and immunoregulatory monocytes in human filarial infections," Journal of Infectious Diseases, vol. 199, no. 12, pp. 1827-1837, 2009.

[72] A. L. Scott, "The alternatively activated human-redux," Journal of Infectious Diseases, vol. 199, no. 12, pp. 1723-1725, 2009.
[73] M. Munder, F. Mollinedo, J. Calafat, et al., "Arginase I is constitutively expressed in human granulocytes and participates in fungicidal activity," Blood, vol. 105, no. 6, pp. 2549-2556, 2005.

[74] M. Munder, H. Schneider, C. Luckner, et al., "Suppression of T-cell functions by human granulocyte arginase," Blood, vol. 108, no. 5, pp. 1627-1634, 2006.

[75] P. Kropf, D. Baud, S. E. Marshall, et al., "Arginase activity mediates reversible $\mathrm{T}$ cell hyporesponsiveness in human pregnancy," European Journal of Immunology, vol. 37, no. 4, pp. 935-945, 2007.

[76] V. Bronte and P. Zanovello, "Regulation of immune responses by L-arginine metabolism," Nature Reviews Immunology, vol. 5, no. 8, pp. 641-654, 2005.

[77] M. Modolell, B. S. Choi, R. O. Ryan, et al., "Local suppression of $\mathrm{T}$ cell responses by arginase-induced L-arginine depletion in nonhealing leishmaniasis," PLoS Neglected Tropical Diseases, vol. 3, no. 7, article e480, 2009.

[78] Y. Latchman, C. R. Wood, T. Chernova, et al., "PD-L2 is a second ligand for PD-1 and inhibits T cell activation," Nature Immunology, vol. 2, no. 3, pp. 261-268, 2001.

[79] Y. E. Latchman, S. C. Liang, Y. Wu, et al., "PD-L1-deficient mice show that PD-L1 on T cells, antigen-presenting cells, and host tissues negatively regulates T cells," Proceedings of the National Academy of Sciences of the United States of America, vol. 101, no. 29, pp. 10691-10696, 2004.

[80] P. Smith, C. M. Walsh, N. E. Mangan, et al., "Schistosoma mansoni worms induce anergy of $\mathrm{T}$ cells via selective upregulation of programmed death ligand 1 on macrophages," The Journal of Immunology, vol. 173, no. 2, pp. 1240-1248, 2004.

[81] F. Kierszenbaum, M. B. Sztein, and L. A. Beltz, "Decreased human IL-2 receptor expression due to a protozoan pathogen," Trends in Immunology, vol. 10, no. 4, pp. 129-131, 1989.

[82] Y. Liu, Y. Yu, S. Yang, et al., "Regulation of arginase i activity and expression by both PD-1 and CTLA-4 on the myeloidderived suppressor cells," Cancer Immunology, Immunotherapy, vol. 58, no. 5, pp. 687-697, 2009.

[83] J. Oberlies, C. Watzl, T. Giese, et al., "Regulation of NK cell function by human granulocyte arginase," The Journal of Immunology, vol. 182, no. 9, pp. 5259-5267, 2009.

[84] I. M. Corraliza, M. Modolell, E. Ferber, and G. Soler, "Involvement of protein kinase A in the induction of arginase in murine bone marrow-derived macrophages," Biochimica et Biophysica Acta, vol. 1334, no. 2-3, pp. 123-128, 1997.

[85] C.-I. Chang, B. Zoghi, J. C. Liao, and L. Kuo, "The involvement of tyrosine kinases, cyclic AMP/protein kinase A, and p38 mitogen-activated protein kinase in IL-13-mediated arginase I induction in macrophages: its implications in IL-13-inhibited nitric oxide production," The Journal of Immunology, vol. 165, no. 4, pp. 2134-2141, 2000.

[86] I. Haffner, D. Teupser, L. M. Holdt, J. Ernst, R. Burkhardt, and J. Thiery, "Regulation of arginase-1 expression in macrophages by a protein kinase A type I and histone deacetylase dependent pathway," Journal of Cellular Biochemistry, vol. 103, no. 2, pp. 520-527, 2008.

[87] C. C. Stempin, V. V. Garrido, L. R. Dulgerian, and F. M. Cerbán, "Cruzipain and SP600125 induce p38 activation, alter $\mathrm{NO}$ /arginase balance and favor the survival of Trypanosoma cruzi in macrophages," Acta Tropica, vol. 106, no. 2, pp. 119127, 2008.

[88] A.-L. Pauleau, R. Rutschman, R. Lang, A. Pernis, S. S. Watowich, and P. J. Murray, "Enhancer-mediated control of 
macrophage-specific arginase I expression," The Journal of Immunology, vol. 172, no. 12, pp. 7565-7573, 2004.

[89] R. Rutschman, R. Lang, M. Hesse, J. N. Ihle, T. A. Wynn, and P. J. Murray, "Cutting edge: Stat6-dependent substrate depletion regulates nitric oxide production," The Journal of Immunology, vol. 166, no. 4, pp. 2173-2177, 2001.

[90] K. C. El Kasmi, J. E. Qualls, J. T. Pesce, et al., “Tolllike receptor-induced arginase 1 in macrophages thwarts effective immunity against intracellular pathogens," Nature Immunology, vol. 9, no. 12, pp. 1399-1406, 2008. 

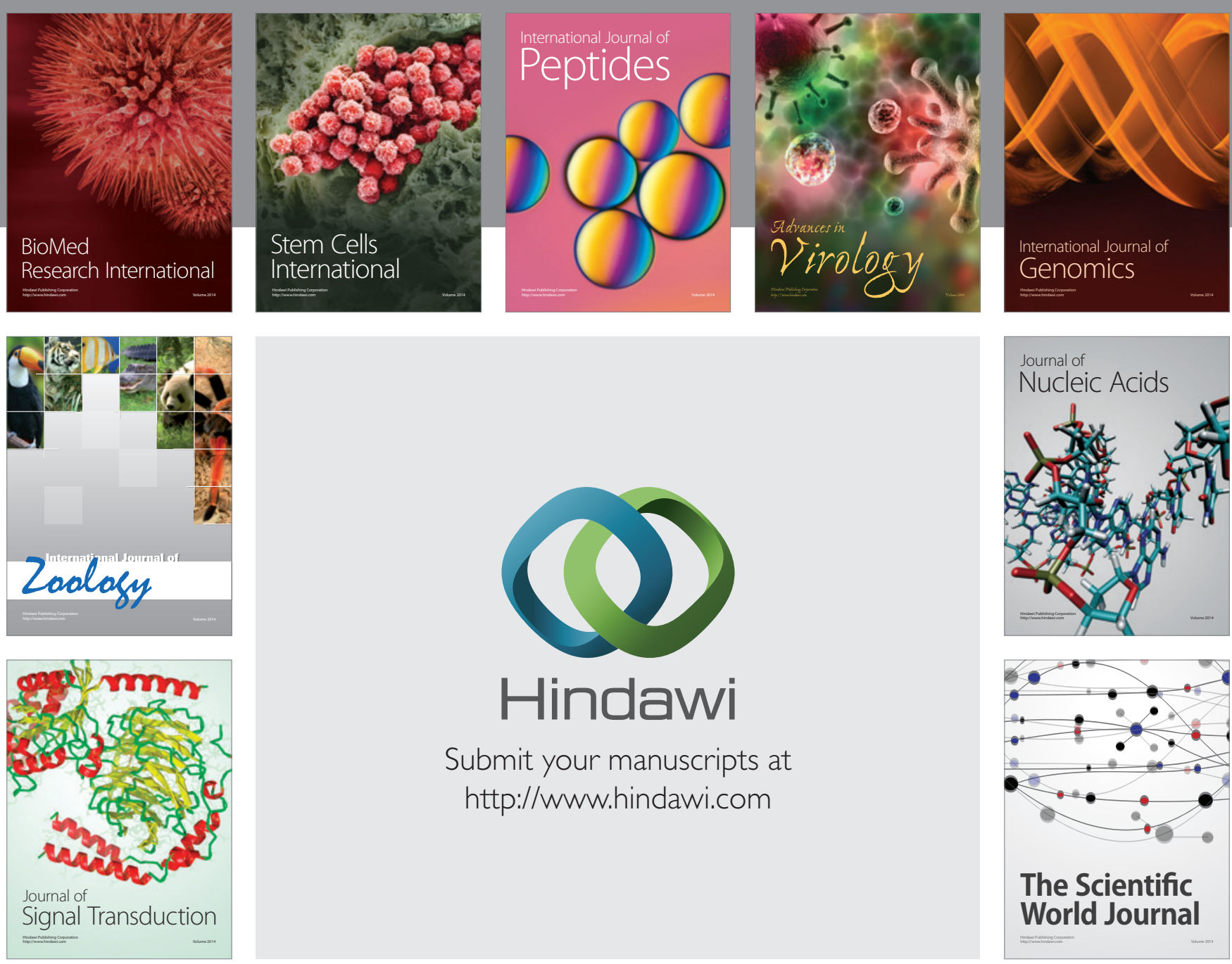

Submit your manuscripts at

http://www.hindawi.com
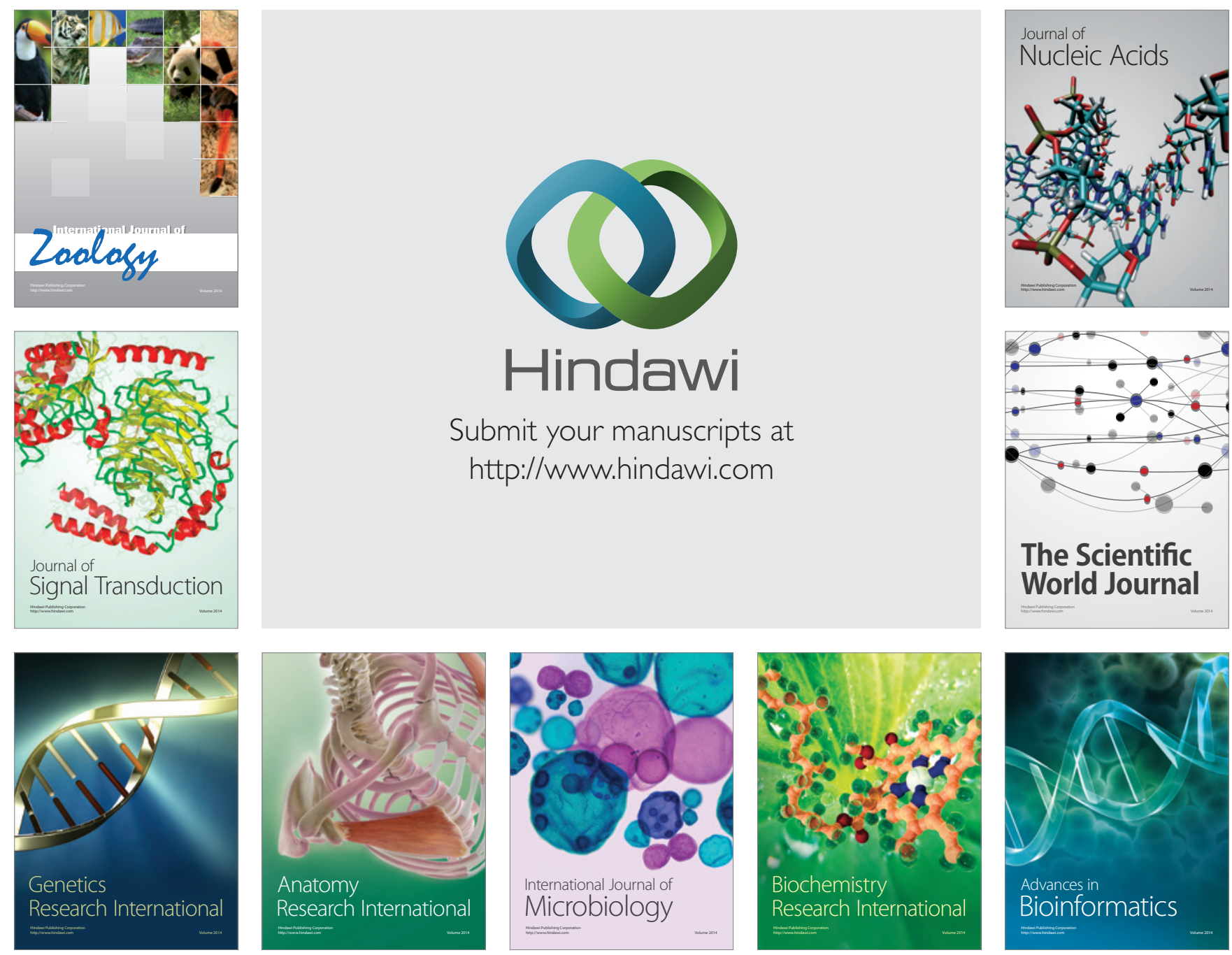

The Scientific World Journal
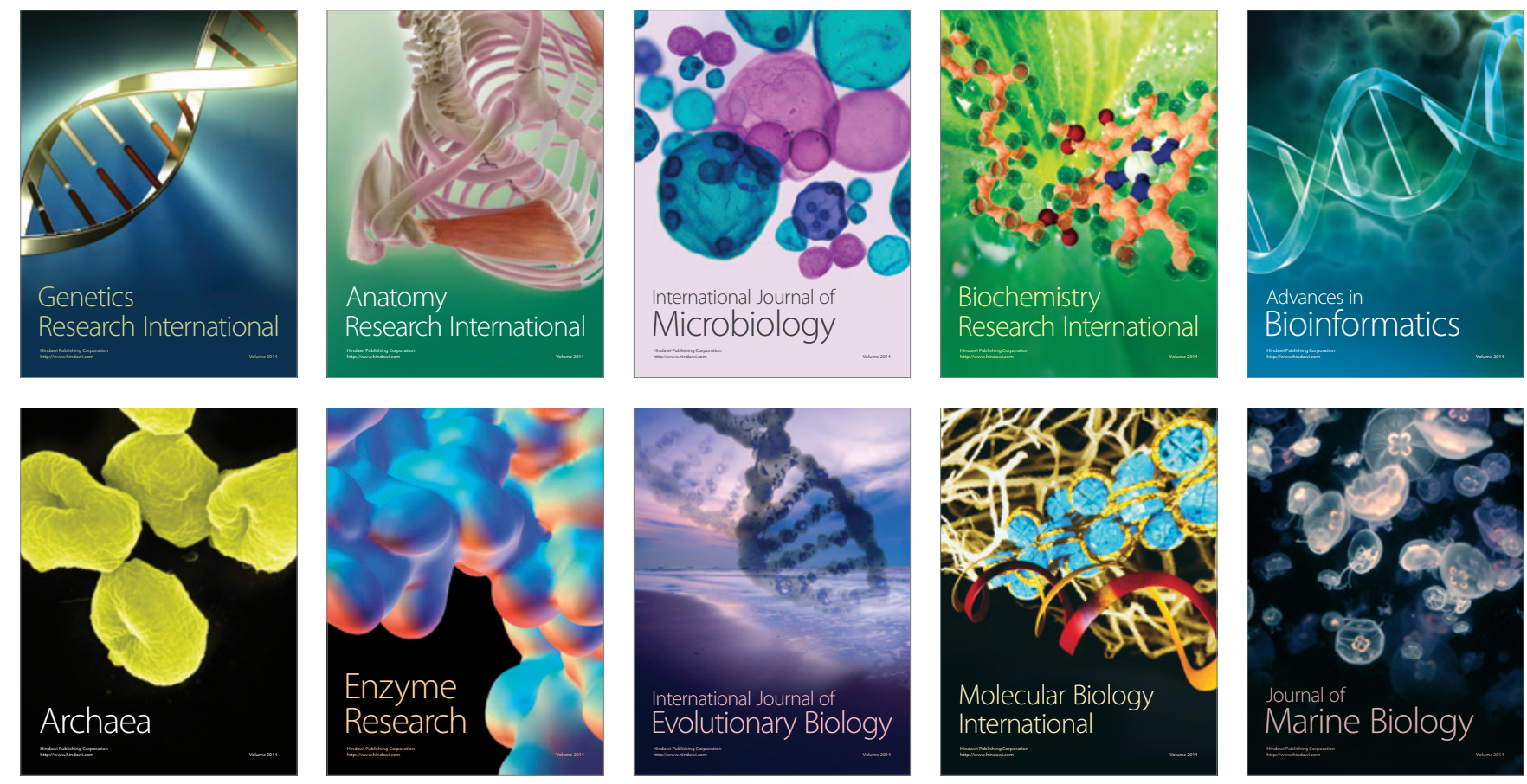